\title{
Analyzing the effect of dilatation on the velocity gradient tensor using a model problem
}

\author{
M. Gonzalez ${ }^{1} \mathbb{D}$
}

Received: 31 March 2020 / Accepted: 15 September 2020 / Published online: 8 October 2020

(c) Springer Nature Switzerland AG 2020

\begin{abstract}
The effect of variable mass density on the velocity gradient tensor is addressed by means of a model problem. An equation system for both the velocity gradient and the pressure Hessian tensor is solved assuming a realistic expansion rate. The model results show the evolution of the velocity gradient tensor as the density front is approached and are relevant to the physics of flame fronts.
\end{abstract}

Keywords Variable mass density · Velocity gradient tensor · Pressure Hessian · Strain properties · Flame fronts

\section{Introduction}

The influence of local mass density variations upon the properties of the velocity gradient tensor is especially significant in compressible flows or in reacting flows with heat release. Intensity and orientation of both strain and vorticity may be altered, which eventually plays on the growth rate and alignment of scalar gradients. Through the velocity gradient, mass density gradients may thus influence the mixing process, a phenomenon addressed in compressible turbulence [1,2] and in turbulent flames [3-5].

Such indirect effects often stem from an intricate interaction. For instance, there is now some evidence that, to a large extent, the small-scale physics of turbulent flames is governed by the interplay of the respective gradients of velocity, concentration, and mass density. Explaining the resulting phenomena may thus require, as a first step, analyzing each underlying mechanism separately. The present work is based on this kind of approach.

The basic model problem is the evolution of the velocity gradient tensor undergoing a given expansion rate. This is a one-way coupling in which heat release, for instance, is forced in a restricted flow region, and subsequently affects the velocity gradient properties. The equation system for the velocity gradient tensor, including the enhanced homogenized Euler equation (EHEE) model of Suman and Girimaji [6] for the pressure Hessian tensor, is solved in a two-dimensional Euler flow (Sect. 2). The evolution of strain structure is analyzed for large and low values of the density ratio (Sect. 3).

\section{Model problem}

In an Euler flow, the evolution of the velocity gradient tensor, $A=\nabla u$, is described by the following equation:

$$
\frac{\mathrm{D} A_{i j}}{\mathrm{D} t}=-A_{i \alpha} A_{\alpha j}-\Pi_{i j}
$$

where the $\Pi_{i j}$ 's are the components of the pressure Hessian tensor, $\Pi=\nabla[(\nabla p) / \rho]$, with $p$ and $\rho$ being, respectively, the pressure and the mass density.

In the two-dimensional case, Eq. (1) can be expressed by a four-equation system:

M. Gonzalez, michel.gonzalez@coria.fr| ${ }^{1}$ CNRS, UMR 6614 CORIA, Site universitaire du Madrillet, 76801 Saint-Etienne du Rouvray, France. 
$\frac{\mathrm{D} \sigma_{n}}{\mathrm{D} t}=-\delta \sigma_{n}+\Pi_{22}-\Pi_{11}$

$\frac{\mathrm{D} \sigma_{s}}{\mathrm{D} t}=-\delta \sigma_{s}-\Pi_{12}-\Pi_{21}$

$\frac{\mathrm{D} \omega}{\mathrm{D} t}=-\delta \omega+\Pi_{12}-\Pi_{21}$

$\frac{\mathrm{D} P}{\mathrm{D} t}=-\frac{1}{2}\left(\sigma^{2}-\omega^{2}+P^{2}\right)-\Pi_{11}-\Pi_{22}$

where $\delta(t)$ is the expansion-or dilatation-rate, $\delta(t)=-1 / \rho \cdot \mathrm{D} \rho / \mathrm{D} t, \sigma_{n}=A_{11}-A_{22}$ and $\sigma_{s}=A_{12}+A_{21}$ are, respectively, the normal and shear components of strain, $\sigma=\left(\sigma_{n}^{2}+\sigma_{s}^{2}\right)^{1 / 2}$ is the strain intensity, $\omega=A_{21}-A_{12}$ is the vorticity, and $P=A_{11}+A_{22}$ is the velocity divergence which - as a result of mass conservation-coincides with the dilatation rate: $P \equiv \delta$.

The model problem is based on assuming the expansion rate as:

$\delta(c)=4 \delta_{m} c(1-c)$,

with $c(t)=\left(\rho_{o} / \rho(t)-1\right) /\left(\rho_{o} / \rho_{\infty}-1\right)$ where $\rho_{o} \equiv \rho(0)$, and $\rho_{\infty} \equiv \lim _{t \rightarrow \infty} \rho(t)$; the density ratio is defined by $\rho_{o} / \rho_{\infty}$. The parabolic function modeling $\delta(c)$ is inspired from numerical simulation data for the velocity divergence across a flame front [7]. From the definitions of $c(t)$ and $\delta(t)$ :

$\frac{\mathrm{D} c}{\mathrm{D} t}=\left(c+\frac{1}{q}\right) \delta$,

with $q=\rho_{o} / \rho_{\infty}-1$. In this study, we state $\delta_{m}=q$, with $q>0$, which means $\rho_{\infty}<\rho_{o}$-and $\delta>0$-as a result, for instance, of heat release. Note that from the approach of Tien and Matalon [8] $\delta \simeq q / \tau_{f}$ in the reaction zone of a flame front, where $\tau_{f}$ is the flame timescale. Stating $\delta_{m}=q$ thus comes to normalize $\delta_{m}$ by $1 / \tau_{f}$. This choice is convenient, for it makes $\delta(c)$ depend on a single parameter. Figure 1 shows $c(t)$ and $\delta(t)$ for $q=5$ and $q=1$.

The evolution of the velocity tensor is computed from Eqs. (2)-(4), with the EHEE modeled equation for $\Pi_{12}, \Pi_{21}$, and $\Pi_{22}[6]$ :

$\frac{\mathrm{D} \Pi_{i j}}{\mathrm{D} t}=-A_{\alpha j} \Pi_{i \alpha}-A_{\alpha i} \Pi_{\alpha j}-(n-1) A_{\alpha \alpha} \Pi_{i j}$

where $n$ is the ratio of specific heats, while component $\Pi_{11}$ is computed from Eq. (5) with $P$ derived from Eqs. (6) and (7).

A study spanning a range of initial conditions is not within the scope of this work. As a first step, the physical relevance of the model is checked with a single set of initial conditions,

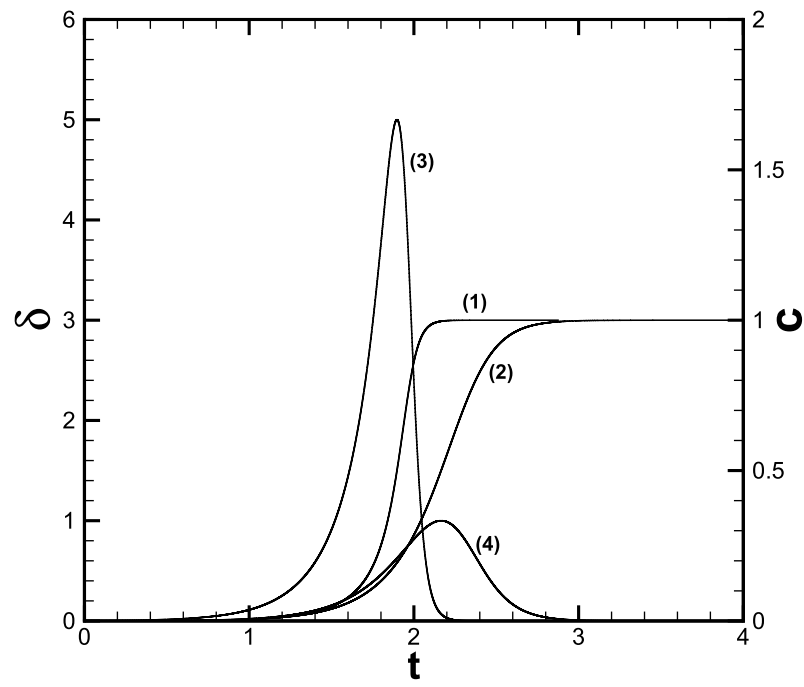

Fig. 1 Evolution of $c$ and $\delta$; (1) $c(t)$ for $q=5$; (2) for $q=1$; (3) $\delta(t)$ for $q=5$; (4) for $q=1$

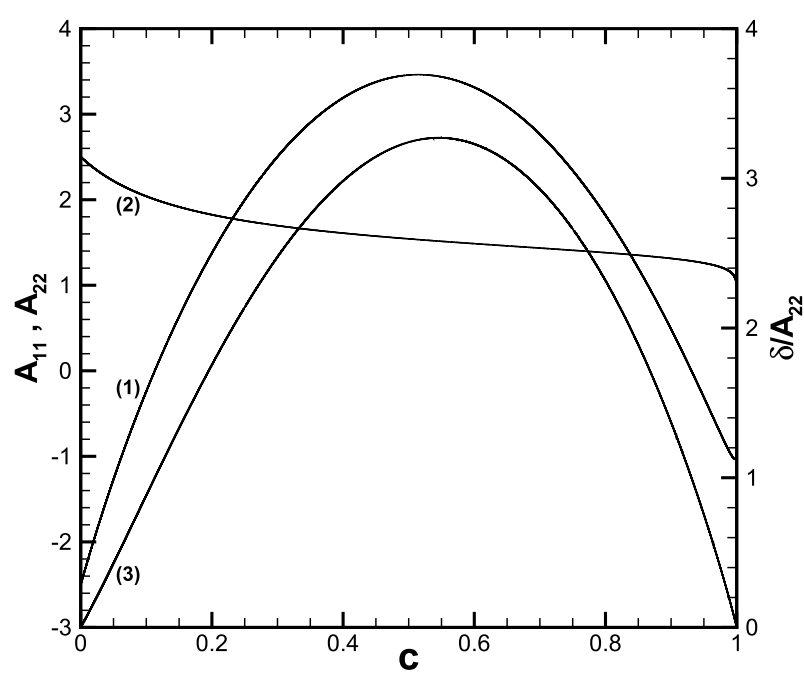

Fig. 2 Diagonal components of $\mathrm{A},(1) A_{11^{\prime}}$ (2) $A_{22^{\prime}}$ (3) ratio $\delta / A_{22^{\prime}}$, versus $c$, for $q=5$

namely: $c(0)=10^{-4}, \sigma_{n}(0)=-\delta_{m}, \sigma_{s}(0)=0.1\left|\sigma_{n}(0)\right|$, $\omega(0)=\sigma(0)$, together with isotropy of tensor $\Pi$, namely $\Pi_{12}(0)=\Pi_{21}(0)=0$, and $\Pi_{11}(0)$ and $\Pi_{22}(0)$ derived from Eq. (5) at $t=0$, with $\Pi_{11}(0)=\Pi_{22}(0)$.

In Fig. $2, A_{11}, A_{22}$ and $\delta / A_{22}$ are plotted against $c$, for $q=5$. Interestingly, the behavior shown in Fig. 2 is akin to the structure of normal strain $\left(a_{N} \equiv A_{11}\right)$ and tangential strain $\left(a_{T} \equiv A_{22}\right)$ across a flame front $[7,9]$. 


\section{Effect of dilatation on strain structure}

The evolution of strain tensor properties, namely strain eigenvalues, $\lambda_{1}=(-\sigma+\delta) / 2$ and $\lambda_{2}=(\sigma+\delta) / 2$ [10] as well as orientation of the strain eigenvectors, $e_{1}$ and $e_{2}$, is examined for both $q=5$ (large density ratio) and $q=1$ (low density ratio ) with the same latter initial conditions.

\subsection{Large density ratio}

Because it determines the sign of the lowest strain eigenvalue, the ratio of dilatation rate to strain intensity, $\delta / \sigma$, is a significant parameter of the dynamics of a scalar gradient in a non-solenoidal flow [10]. As shown in Fig. 3, dilatation makes the smallest eigenvalue, $\lambda_{1}$, positivewhich thus means two extensional strain directionsover most of the $c$-range, where $\delta / \sigma>1$. It is only at the edges ( $c<0.12$, and $c>0.92$ ), where $\delta / \sigma<1$, that $\lambda_{1}<0$, which thus leads to one compressional and one extensional strain directions.

The orientation of strain eigenvectors is shown by $\Phi=\arctan \left(\sigma_{n} / \sigma_{s}\right) / 2-\pi / 4$, the angle between axis $\mathrm{x}_{1}$ and the direction of the largest strain, $\mathrm{e}_{2}$ (Fig. 4). For small $c, \Phi<-\pi / 4$, which means that direction $\mathrm{x}_{1}$ mostly undergoes the influence of the smallest strain. As $c$ increases, counterclockwise rotation of strain axes brings the direction of the largest strain near $\mathrm{x}_{1}$, and this orientation is hold all over the intermediate $c$-range. As $c$ reaches the upper range, rotation of strain axes is reversed, and the direction of the lowest strain comes back close to $x_{1}$. These changes in strain axes orientation, in particular, alignment of the largest strain with the

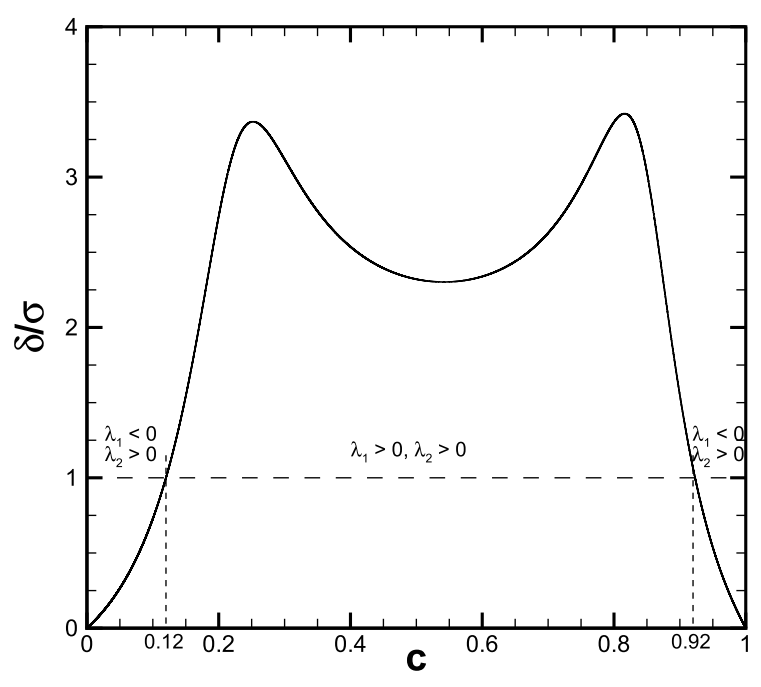

Fig. 3 Ratio $\delta / \sigma$ and sign of strain eigenvalues versus $c ; q=5$

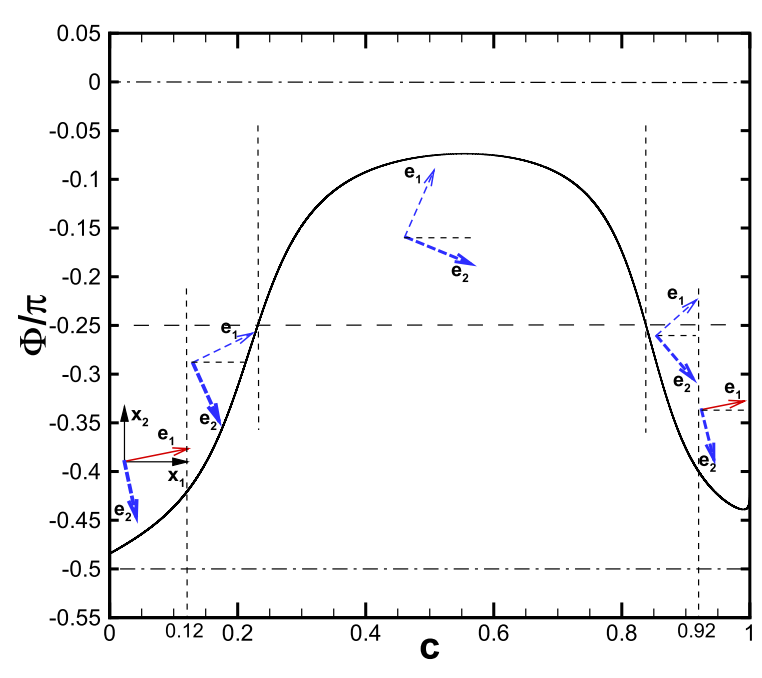

Fig. 4 Angle $\Phi$ between $x_{1}$ and the direction of largest strain, $e_{2}$, versus $c ; q=5$; a solid arrow indicates compressional strain, while a dashed arrow indicates extensional strain; direction of the largest strain is shown by a bold dashed arrow

direction of anisotropy, are consistent with the evolution of strain approaching a flame front $[5,11]$.

In this two-dimensional Euler flow, rotation of strain principal axes is promoted by anisotropy of the pressure Hessian tensor [12]. The rotation rate of strain eigenvectors is indeed given by $\Omega=2 \mathrm{D} \Phi / \mathrm{D} t=\sigma^{-2}\left(\sigma_{s} \mathrm{D} \sigma_{n} / \mathrm{D} t-\sigma_{n} \mathrm{D} \sigma_{s} / \mathrm{D} t\right)$, and then, from Eqs. (2) and (3), $\Omega=\sigma^{-2}\left[\sigma_{s}\left(\Pi_{22}-\Pi_{11}\right)+\sigma_{n}\left(\Pi_{12}+\Pi_{21}\right)\right]$. Figure 5 clearly shows the anisotropy of $\Pi$ revealed by $\Pi_{11}$ prevailing over the other components, and the resulting rotation rate, $\Omega$.

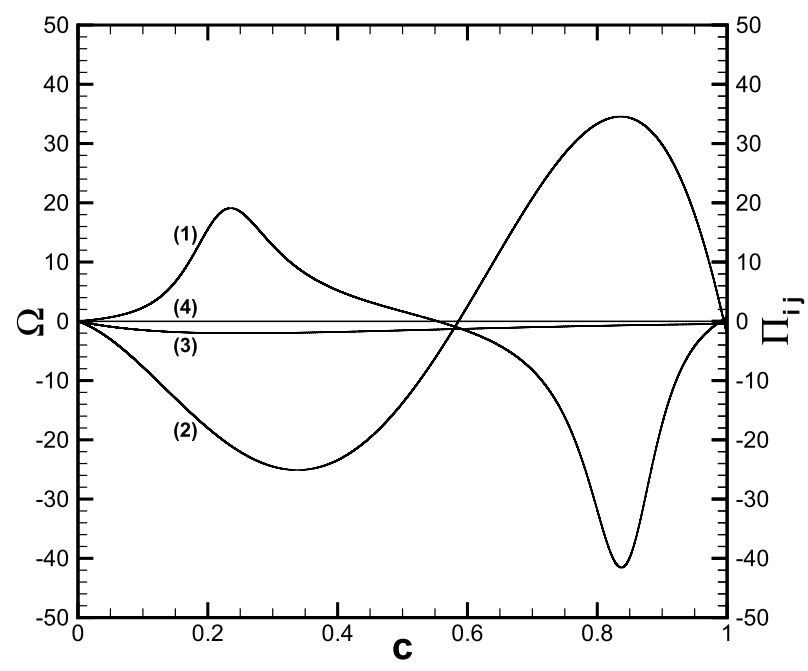

Fig. 5 Rotation rate of strain principal axes, $\Omega$, and components of pressure Hessian tensor, $\Pi_{i j}$, versus $c ; q=5 ;$ (1) $\Omega$; (2) $\Pi_{11} ;(3) \Pi_{12}$ and $\Pi_{21} ;(4) \Pi_{22}$ 


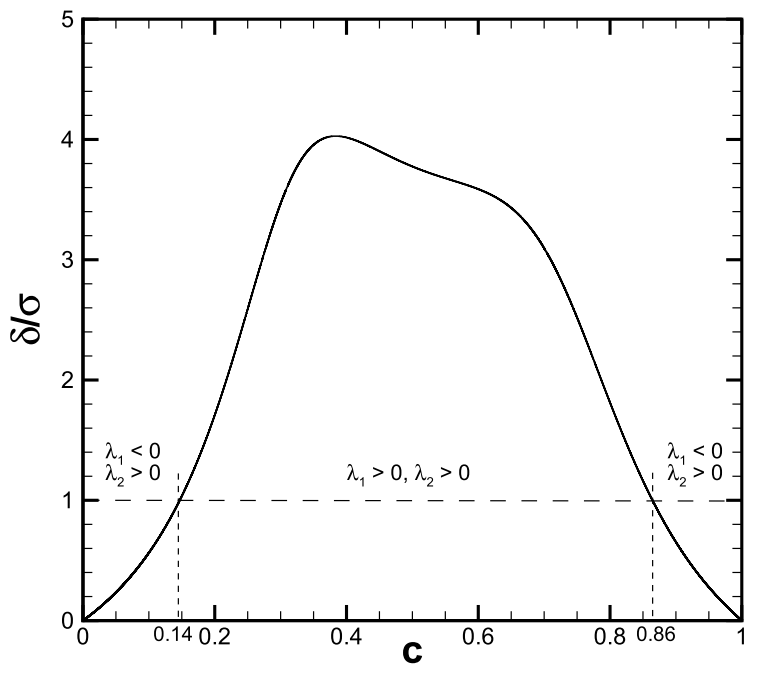

Fig. 6 Ratio $\delta / \sigma$ and sign of strain eigenvalues versus $c ; q=1$

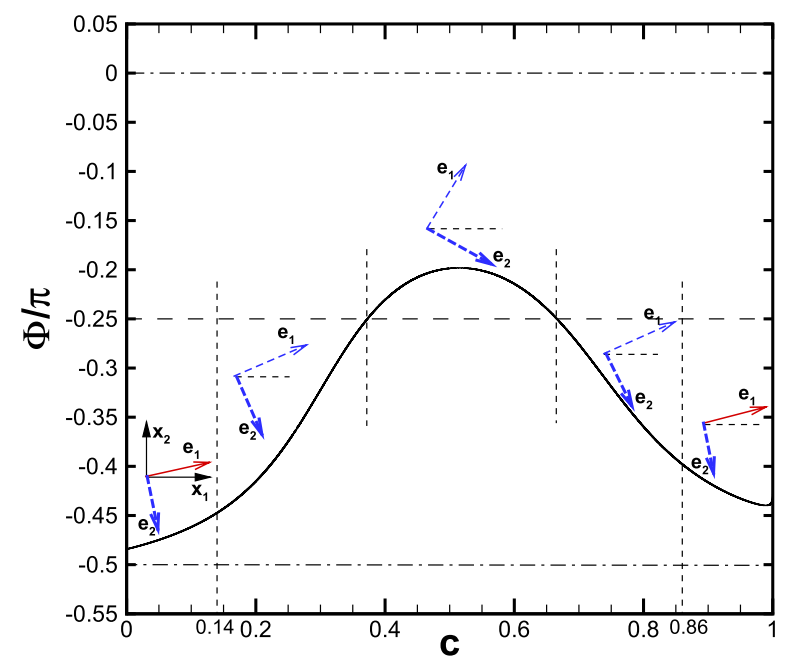

Fig. 7 Angle $\Phi$ between $x_{1}$ and the direction of largest strain, $e_{2}$, versus $c ; q=1$; a solid arrow indicates compressional strain, while a dashed arrow indicates extensional strain; direction of the largest strain is shown by a bold dashed arrow

\subsection{Low density ratio}

Similar features of strain evolution are retrieved for $q=1$ with, however, a lesser influence of expansion rate. The lowest strain eigenvalue again gets positive for intermediate $c$ values as expansion rate exceeds strain intensity, but this time over a somewhat shorter range (Fig. 6).

The influence of density ratio is more obvious in strain axes orientation (Fig. 7). The direction of the largest strain comes much less close to $x_{1}$ over the intermediate $c$-range, and the latter is more narrow as well. This directly results from a weaker rotation rate, $\Omega$, for $q=1$ (not shown). Indeed the lower $q$, the smaller the respective magnitudes of strain components, $\sigma_{n}$ and $\sigma_{s}$ and of anisotropic terms, $\Pi_{22}-\Pi_{11}$ and $\Pi_{12}+\Pi_{21}$; and the level of $\sigma^{-2}$-greater for $q=1$ than for $q=5$-is not enough to balance this difference.

\section{Conclusion}

The mechanisms underlying the influence of dilatation on the velocity gradient tensor can be reliably addressed with a model problem. Assuming a likely evolution of the expansion rate, the solution of an equation system for the components of the velocity gradient and of the pressure Hessian includes a number of features regarding the evolution of the dynamic field as a density front is approached.

The ratio of dilatation rate to strain intensity, a critical parameter in the variable-mass-density kinematics of scalar gradient, is derived. More specifically, the evolution of normal and tangential strains is reminiscent of strain structure at the crossing of a flame front. Finally, the pressure Hessian anisotropy resulting from forcing the expansion rate promotes the rotation of strain principal axes, which subsequently aligns the largest strain with the direction of anisotropy, a result relevant to questions at issue in the physics of flames. Extension of the approach to the threedimensional case as well as to the coupling of the velocity gradient dynamics with the physics of a reacting scalar gradient is a work in progress.

\section{Compliance with ethical standards}

Conflict of interest On behalf of all authors, the corresponding author states that there is no conflict of interest.

\section{References}

1. Danish M, Suman S, Girimaji SS (2016) Influence of flow topology and dilatation on scalar mixing in compressible turbulence. $J$ Fluid Mech 793:633-655

2. Boukharfane R, Bouali Z, Mura A (2018) Evolution of scalar and velocity dynamics in planar shock-turbulence interaction. Shock Waves 28:1117-1141

3. Swaminathan N, Grout RW (2006) Interaction of turbulence and scalar fields in premixed flames. Phys Fluids 18:045102

4. Dopazo C, Cifuentes L (2016) The physics of scalar gradients in turbulent premixed combustion and its relevance to modeling. Combust Sci Technol 188:1376-1397

5. Zhao S, Er-ray A, Bouali Z, Mura A (2018) Dynamics and kinematics of the reactive scalar gradient in weakly turbulent premixed flames. Combust Flame 198:436-454

6. Suman S, Girimaji SS (2011) Dynamical model for velocitygradient evolution in compressible turbulence. J Fluid Mech 683:289-319 
7. Dopazo C, Cifuentes L, Martin J, Jimenez C (2015) Strain rates normal to approaching iso-scalar surfaces in a turbulent premixed flame. Combust Flame 162:1729-1736

8. Tien JH, Matalon M (1991) On the burning velocity of stretched flames. Combust Flame 84:238-248

9. Chakraborty N, Rogerson JW, Swaminathan N (2010) The scalar gradient alignment statistics of flame kernels and its modelling implications for turbulent premixed combustion. Flow Turbul Combust 85:25-55

10. Gonzalez M, Paranthoën $P$ (2011) Effect of variable mass density on the kinematics of the scalar gradient. Phys Fluids 23:075107

11. Steinberg AM, Driscoll JF, Swaminathan N (2012) Statistics and dynamics of turbulence-flame alignment in premixed combustion. Combust Flame 159:2576-2588
12. Lapeyre G, Klein P, Hua BL (1999) Does the tracer gradient align with strain eigenvectors in 2D turbulence? Phys Fluids 11:3729-3737

Publisher's Note Springer Nature remains neutral with regard to jurisdictional claims in published maps and institutional affiliations. 